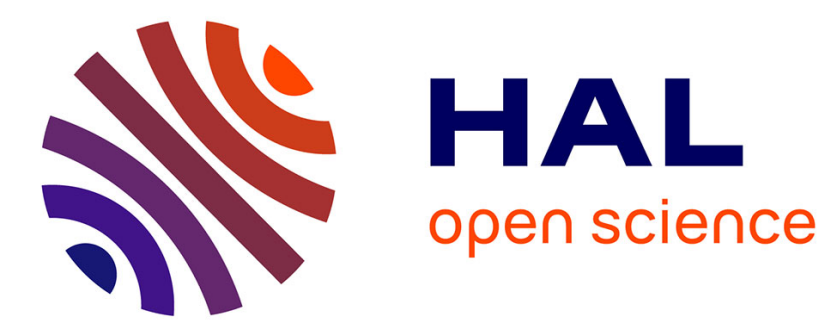

\title{
Prescriptive and descriptive obligations in dynamic epistemic deontic logic
}

\author{
Guillaume Aucher, Guido Boella, Leendert van Der Torre
}

\section{To cite this version:}

Guillaume Aucher, Guido Boella, Leendert van Der Torre. Prescriptive and descriptive obligations in dynamic epistemic deontic logic. AICOL - AI Approaches to the Complexity of Legal Systems - 2009, Dec 2009, Rotterdam, Netherlands. pp.150-161. inria-00556080v2

\section{HAL Id: inria-00556080 \\ https://inria.hal.science/inria-00556080v2}

Submitted on 8 Sep 2013

HAL is a multi-disciplinary open access archive for the deposit and dissemination of scientific research documents, whether they are published or not. The documents may come from teaching and research institutions in France or abroad, or from public or private research centers.
L'archive ouverte pluridisciplinaire HAL, est destinée au dépôt et à la diffusion de documents scientifiques de niveau recherche, publiés ou non, émanant des établissements d'enseignement et de recherche français ou étrangers, des laboratoires publics ou privés. 


\title{
Prescriptive and Descriptive Obligations in Dynamic Epistemic Deontic Logic
}

\author{
Guillaume Aucher ${ }^{1}$, Guido Boella ${ }^{2}$, and Leendert van der Torre ${ }^{1}$ \\ 1 Computer Science and Communications Department - University of Luxembourg, \\ 6, rue Richard Coudenhove - Kalergi L-1359 Luxembourg \\ \{guillaume.aucher, leon. vandertorre\}@uni.lu \\ 2 Dipartimento di Informatica - Università di Torino \\ Cso Svizzera 185, I-10149 Italy \\ guido@di.unito.it
}

\begin{abstract}
Normative sentences can be used to change or to describe the normative system, known as prescriptive and descriptive obligations respectively. In applications of deontic logic it is important to distinguish these two uses of normative sentences. In this paper we show how they can be distinguished and analysed in a Dynamic Epistemic Deontic Logic.
\end{abstract}

\section{Introduction}

Alchourrón and Bulygin [12] discuss the possibility of a logic of norms, which they distinguish from the logic of normative propositions. Roughly, the distinction between norms and normative propositions is that the former are prescriptive whereas the latter are descriptive. In the second sense, the sentence 'it is obligatory to keep right on the streets' is a description of the fact that a certain normative system contains an obligation to keep right on the streets. In the first sense this statement is the obligation of traffic law itself. This distinction goes back to an old philosophical problem discussed by Von Wright [1415], who was hesitant to call deontic formulas 'logical truths,' because "it seems to be a matter of extra-logical decision when we shall say that 'there are' or 'are not' such and such norms." Makinson [10] turns this fundamental problem into the central challenge in deontic logic, which led to new developments over the past decade such as deontic update semantics [13, input/output logic [1], imperative based deontic logic [8], and more.

The relevance of the distinction between prescriptive and descriptive obligations, and the related fundamental problem that norms do not have truth values, is not only theoretical and conceptual, but it has important practical implications for normative multi-agent systems. For example, assume that you ask the librarian of your university to get a journal paper available on the Springer web-site. The library has a list of journals published by Springer containing the journals for which the library has free access (either a journal of this list is on open access or the university subscribed to it). This list is updated every month but the librarian did not check it for some time, and so he does not know if the

P. Casanovas et al. (Eds.): AICOL Workshops 2009, LNAI 6237, pp. $150-161,2010$.

(C) Springer-Verlag Berlin Heidelberg 2010 
requested journal is on the list, although he should actually know it. He then logs on the journal and tries to download the paper. We consider two kinds of events corresponding to two different uses of obligations.

Descriptive use. The Springer web-site informs the librarian that he should not pay to download any paper of this journal. As a result, the librarian now knows that this journal was on the list of journals for which the library has free access.

Prescriptive use. The Springer web-site refuses to download the file. However, after the librarian make a new contract with Springer, the web-site declares that he should not pay to download any paper of this journal since for the contract the journal is now on free of charge for the library.

As we see in this example, modeling the distinction between prescriptive and descriptive use of norms is useful for designing normative multi-agent systems. In this paper we therefore study the following question:

- How can the distinction between prescriptive and descriptive use of obligation be captured in a dynamic epistemic deontic logic?

We are going to introduce a general and expressive Dynamic Epistemic Deontic Logic combining a simplified version of Castañeda's deontic logic [6] with a dynamic epistemic logic. This logic can express the conditional character of norms, study the interaction between epistemic and deontic notions, and model norm dynamics. These three features are fundamental to normative systems (and the representation of legal systems), and also to multi-agent systems as far as those are intended to model real life. First, in normative systems it is necessary to express realistic regulations, which have a conditional character. Secondly, communication is an essential part of normative and multi-agent systems, and this raises the issue of what it is permitted, prohibited or obliged to know by agents, for example, when modelling privacy regulations. Thirdly, normative multi-agent systems have a dynamic character, as witnessed by the second definition of normative multi-agent system provided in [5]. These last two issues, communication and dynamics are both useful for distinguishing when existing norms are communicated from the case where a norm is actually put into existence by a declaration, i.e., Alchourrón's distinction between the descriptive and prescriptive use of norms.

The paper is structured as follow. In Section 2 we introduce an epistemic deontic logic. In Section 3 we extend the logic by introducing update operators which change beliefs and norms, and in Section 4 we show how the distinction between descriptive and prescriptive norms can be made in our logic if we map this distinction to the context of agent communication.

\section{Epistemic Deontic Logic (EDL)}

\subsection{Propositions vs. Practitions}

Because of its clear and natural distinction between propositions and practitions and its modal-like character, the well known deontic logic of Castañeda [6] 
lends itself very well to the introduction of an epistemic logic. Starting from a linguistic analysis, the insight of Castañeda's well known approach to deontic logic is to acknowledge the grammatical duality of expressions depending whether they are within or without the scope of deontic operators [6]. This leads him formally to introduce two sets of propositional letters: $\Phi^{\phi}$ called propositions which cannot alone be the foci of deontic operators, unlike $\Phi^{\alpha}$ called practitions. The former are usually expressed grammatically in the indicative form and the latter are usually expressed grammatically in the infinitive/subjunctive form. For example, "the librarian does not pay to access a journal" in the indicative form is a proposition, but the same sentence in "it is obligatory for the librarian not to pay to access a journal" in subjunctive/infinitive form is a practition. $\mathrm{He}$ then defines more general propositions $\mathcal{L}_{D L}^{\phi}$ and practitions $\mathcal{L}_{D L}^{\alpha}$ as follows.

$$
\begin{gathered}
\mathcal{L}_{D L}^{\phi}: \phi::=p|\phi \wedge \phi| \neg \phi \mid O \alpha \\
\mathcal{L}_{D L}^{\alpha}: \alpha::=\beta|\neg \alpha| \alpha \wedge \alpha|\alpha \wedge \phi| \phi \wedge \alpha
\end{gathered}
$$

where $\beta$ ranges over $\Phi^{\alpha}$ and $p$ over $\Phi^{\phi}$. We define the language $\mathcal{L}_{D L}=\mathcal{L}_{D L}^{\phi} \cup \mathcal{L}_{D L}^{\alpha}$, whose formulas are generally denoted $\phi^{*}$. In the sequel, $P \alpha$ is an abbreviation for $\neg O \neg \alpha$. $O \alpha$ reads ' $\alpha$ is obligatory' and $P \alpha$ reads ' $\alpha$ is permitted'.

We now propose a semantics based on modal logic which is equivalent to the one of Castañeda, in the sense that any 'Castañeda'-model [6] can be transformed into a $D L$-model satisfying the same formulas, and vice versa.

Definition 1. A DL-model $M$ is a tuple $M=(W, D, V)$ where $W$ is a nonempty set of possible worlds, $D$ is a serial accessibility relation on $W$ and $V$ is a valuation which assigns to each propositional letter $p^{*} \in \Phi^{\phi} \cup \Phi^{\alpha}$ a subset of $W$, such that for all $w \in W$, all $p \in \Phi^{\phi}$,

$$
V(p) \cap(D(w) \cup\{w\})=D(w) \cup\{w\} \text { or } \emptyset
$$

Let $M=(W, D, V)$ be a $D L$-model, $w \in W$ and $\phi^{*} \in \mathcal{L}_{D L},(M, w)$ is called a pointed $D L$-model. We define $M, w \models \phi^{*}$ inductively as follows.

$$
\begin{array}{lll}
M, w \models p^{*} & \text { iff } & w \in V\left(p^{*}\right) \\
M, w \models \phi^{*} \wedge \psi^{*} & \text { iff } & M, w \models \phi^{*} \text { and } M, w \models \psi^{*} \\
M, w \models \neg \phi^{*} & \text { iff } & \text { not } M, w \models \phi^{*} \\
M, w \models O \alpha & \text { iff } & \text { for all } v \in D(w), M, v \models \alpha .
\end{array}
$$

Condition $(*)$ above ensures formally that conditional norms of the form "it is obligatory that if the librarian knows that a journal is in the list" $O(p \rightarrow \alpha)$ are equivalent to "if the librarian knows that journal is on the free access list then he should not pay" $(p \rightarrow O \alpha)$ :

$$
\models O(p \rightarrow \alpha) \leftrightarrow(p \rightarrow O \alpha)
$$

More generally, condition $(*)$ allows us to show that any deontic formula with practition(s) $\mathcal{L}_{D L}^{\alpha}$ involving proposition(s) is actually equivalent to a deontic formula with 'pure' practitions $\mathcal{L}_{D L}^{\alpha^{\prime}}$, i.e. a formula of $\mathcal{L}_{D L}^{\prime}=\mathcal{L}_{D L}^{\phi} \cup \mathcal{L}_{D L}^{\alpha^{\prime}}$ :

\footnotetext{
${ }^{1}$ A relation $R$ is serial iff $R(w) \neq \emptyset$ for all $w \in W$.
} 


$$
\mathcal{L}_{D L}^{\alpha^{\prime}}: \alpha::=\beta|\neg \alpha| \alpha \wedge \alpha
$$

where $\beta$ ranges over $\Phi^{\alpha}$.

Proposition 1. Let $\phi \in \mathcal{L}_{D L}$. There is $\phi^{\prime} \in \mathcal{L}_{D L}^{\prime}$ such that $\models \phi \leftrightarrow \phi^{\prime}$.

In other words, instead of dealing with formulas of $\mathcal{L}_{D L}$, we could equivalently deal only with formulas of $\mathcal{L}_{D L}^{\prime}$.

\subsection{Adding Beliefs}

Just as practitions are the foci of deontic operators, propositions are dually the foci of knowledge operators, as pointed out by Castañeda [7. An expression $\phi$ in the scope of a belief operator $B \phi$ is always in the indicative form and never in the subjunctive/infinitive form, even if $B \phi$ is in the scope of a deontic operator $O$. We extend Castañeda [7]'s intuition to the context of epistemic permissions and obligations. In a deontic setting the reading of the term knowledge or belief can also be twofold: either as a proposition or as a practition. On the one hand, in the sentence "it is obligatory that the librarian knows / for the librarian to know that he should not pay" the verb 'to know' is the focus of a deontic operator and is in the subjunctive/infinitive form. On the other hand, the sentence "The librarian knows that he should not pay' alone describes a circumstance and the interpretation of the verb 'to know' in the indicative form matches the one usually studied in epistemic logic. The former use of the term knowledge within the scope of a deontic operator is not studied in epistemic logic. For these reasons we enrich the language $\mathcal{L}_{D L}$ with two knowledge modalities, one for propositions and the other for practitions. This yields the following language $\mathcal{L}_{E D L}=\mathcal{L}_{E D L}^{\phi} \cup$ $\mathcal{L}_{E D L}^{\alpha}$ whose formulas are generally denoted $\phi^{*}$.

$$
\begin{gathered}
\mathcal{L}_{E D L}^{\phi}: \phi::=p|\neg \phi| \phi \wedge \phi|B \phi| O \alpha \\
\mathcal{L}_{E D L}^{\alpha}: \alpha::=\beta|\neg \alpha| \alpha \wedge \alpha|\alpha \wedge \phi| \phi \wedge \alpha \mid B^{\prime} \phi
\end{gathered}
$$

where $p$ ranges over $\Phi^{\phi}$ and $\beta$ over $\Phi^{\alpha}$. As argued above we do not allow formulas of the form $B \alpha$ or $B^{\prime} \alpha$ because they are linguistically meaningless, which is actually in line with Castañeda [7]. $B \phi$ reads 'the agent believes $\phi$ '.

Definition 2. An EDL-model $M$ is a tuple $M=\left(W, D, R, R^{\prime}, V\right)$ where $W$ is a non-empty set of possible worlds, $R, R^{\prime}$ and $D$ are accessibility relations on $W$, $D$ being serial, and $V$ is a valuation such that:

for all $w \in W$, all $v, v^{\prime} \in D(w) \cup\{w\},(M, v)$ is RD-bisimilar to $\left(M, v^{\prime}\right) \underline{2}$ $(* *)$

The truth conditions for $B$ and $B^{\prime}$ are given by:

$$
\begin{aligned}
& M, w=B \phi \quad \text { iff } \quad \text { for all } v \in R(w), M, v=\phi \\
& M, w=B^{\prime} \phi \quad \text { iff } \quad \text { for all } v \in R^{\prime}(w), M, v \models \phi
\end{aligned}
$$

$M \models \phi$ if for all $w \in W, M, w \models \phi .(M, w)$ is called a pointed EDL-model.

\footnotetext{
${ }^{2}$ Two pointed models $(M, v)$ and $\left(M^{\prime}, v^{\prime}\right)$ are $R D$-bisimilar if there is a relation on $W \times W^{\prime}$ satisfying the base condition for $\Phi^{\phi}$ and the back and forth conditions for $R$ and $D$ (see Blackburn et al. 4, for details).
} 
Note that condition $(* *)$ is a generalization of condition $(*)$ to the epistemic setting: the worlds of $D(w) \cup\{w\}$ are not only 'propositionally bisimilar' as in $(*)$, but also 'epistemically (and deontically) bisimilar'. Two worlds being propositionally bisimilar intuitively means that they satisfy the same propositional formulas, and two worlds being epistemically (and deontically) bisimilar intuitively means (in a finite model) that they satisfy the same epistemic (and deontic) formulas (see 4] for details). Therefore, our conditions $(*)$ and $(* *)$ somehow intuitively mean that the actual epistemic and propositional context is fixed for a given normative situation (represented by $D(w)$ ).

We do not assume any logical property for our notion of belief (such as consistency or introspection) because it is not really relevant for the topic of this paper. For the same reason, the operator $B$ stands alternatively for knowledge or for belief.

Just as for $\mathcal{L}_{D L}$, we can show that the language $\mathcal{L}_{E D L}$ is actually 'equivalent' to the language $\mathcal{L}_{E D L}^{\prime}=\mathcal{L}_{E D L}^{\phi} \cup \mathcal{L}_{E D L}^{\alpha^{\prime}}$ with 'pure' practitions $\mathcal{L}_{E D L}^{\alpha^{\prime}}$ :

$$
\mathcal{L}_{E D L}^{\alpha^{\prime}}: \alpha::=\beta|\neg \alpha| \alpha \wedge \alpha \mid B^{\prime} \phi
$$

Proposition 2. Let $\phi \in \mathcal{L}_{E D L}$. There is $\phi^{\prime} \in \mathcal{L}_{E D L}^{\prime}$ such that $\models \phi \leftrightarrow \phi^{\prime}$.

In other words, instead of dealing with formulas of $\mathcal{L}_{E D L}$, we could equivalently deal only with formulas of $\mathcal{L}_{E D L}^{\prime}$.

Theorem 1. The semantics of $\mathcal{L}_{E D L}$ is sound and complete with respect to the decidable logic $L_{E D L}$ axiomatized as follows:

$$
\begin{aligned}
& A_{1} \quad \text { All propositional tautologies based on } \Phi^{\phi} \cup \Phi^{\alpha} \\
& A_{2} \vdash(\phi \rightarrow O \alpha) \leftrightarrow O(\phi \rightarrow \alpha) \\
& A_{3} \vdash O \alpha \rightarrow \neg O \neg \alpha \\
& A_{4} \vdash O\left(\alpha \rightarrow \alpha^{\prime}\right) \rightarrow\left(O \alpha \rightarrow O \alpha^{\prime}\right) \\
& A_{5} \vdash B^{*}\left(\phi^{*} \rightarrow \psi^{*}\right) \rightarrow\left(B^{*} \phi^{*} \rightarrow B^{*} \psi^{*}\right) \\
& R_{1} \quad \text { If } \vdash \alpha \text { then } \vdash O \alpha \\
& R_{2} \quad \text { If } \vdash \phi^{*} \text { then } \vdash B^{*} \phi^{*} \\
& R_{3} \text { If } \vdash \phi^{*} \rightarrow \psi^{*} \text { and } \vdash \phi^{*} \text { then } \vdash \psi^{*}
\end{aligned}
$$

where $B^{*}$ stands for $B$ or $B^{\prime}$.

Note that axioms $A_{1}$ to $A_{4}$ and rules $R_{1}$ and $R_{3}$ provide an alternative axiomatization of Castañeda's language $\mathcal{L}_{D L}$.

\subsection{Example}

Our logic can express conditional norms, like Castañeda's deontic logic does (i.e., $\vdash(\phi \rightarrow O \alpha) \leftrightarrow O(\phi \rightarrow \alpha))$. Due to its combination of deontic and epistemic notions, it can also express the knowledge-based obligations of Pacuit and Parikh 12]. But because our combination is quite general, we can also express epistemic norms. 
Example 1 (Journal example). Assume that the librarian does not know whether the journal requested is on the list of journals to which the library has free access $(\neg B \neg J$ InList $\wedge \neg B$ J InList). As a matter of fact, according to the library regulations, he should know whether it is a journal to which the library has free access $\left(n_{1}\right)$. Besides, he should also know that if it is a journal for which the library has free access then he should not to pay to download any paper of this journal $\left(n_{2}\right)$. These two epistemic norms are formalized as follows:

$$
\begin{gathered}
n_{1}=O\left(B^{\prime} \text { JInList } \vee B^{\prime} \neg J \text { InList }\right) \\
n_{2}=O B^{\prime}(\text { JInList } \rightarrow O \neg \text { pay })
\end{gathered}
$$

where JInList stands for 'the Journal requested is in the List of journals for which the library has free access' and pay stands for 'pay Springer to download any paper of the journal'.

This situation is depicted in the EDL-model $M$ of Figure 1, where JInList stands for the proposition 'the journal is in the list of journals for which the library has free access' and pay for the practition 'pay Springer to download any paper of the journal'. The dotted arrows correspond to the deontic accessibility relation $D$ and the plain arrows correspond to accessibility relations $R$ and $R^{\prime}$. Reflexive arrows are omitted, which means that for all $v \in M$, we have that $v \in$ $R(v), v \in R^{\prime}(v)$ and $v \in D(v)$. $w$ corresponds to the actual world. We therefore have $M, w \models(\neg B J$ InList $\wedge \neg B \neg J$ InList $) \wedge O\left(B^{\prime} J\right.$ InList $\vee B^{\prime} \neg J$ InList $)$ : the librarian does not know whether the requested journal is on the list of journals free of charge for the library (some of the $R$ accessible worlds contain $\neg J$ InList). However, he should know whether this is the case (in all $D$ worlds it is possible to access via $R^{\prime}$ either only worlds where pay is true or $\neg$ pay is true. $M, w \models$ $B($ J InList $\rightarrow O \neg$ pay $) \wedge B(\neg$ JInList $\rightarrow(\neg$ Opay $\wedge \neg O \neg$ pay $))$ : the librarian knows that if the journal is in the list then he should not pay to download any paper of the journal (in no world where JInList is true it is possible to access via $D$ a world where pay is true) and he knows that if it is not in the list then he might or might not have to pay to download papers of the journal (in each world where JInList is false, it is not possible to access via $D$ worlds where only pay or $\neg$ pay is true). For example, because the journal is then on limited access and some papers might be available for free whereas some others might not.

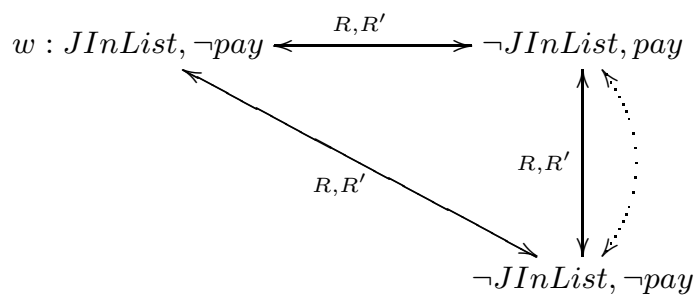

Fig. 1. Journal example 


\section{Dynamic Epistemic Deontic Logic (DEDL)}

\subsection{Changing Norms and Beliefs}

We now want to add dynamics to the picture by means of communicative acts made to the agent. The content of these communicative acts can affect the situation in two ways: either it affects the epistemic realm (represented in a $E D L$-model by the relation $R$ ) or it affects the normative realm (represented in a $E D L$-model by the relations $R^{\prime}$ and $D$ ). This leads us to enrich the language $\mathcal{L}_{E D L}$ with two dynamic operators [ $\left.\phi !\right]$ and [ $\left.\phi^{*} ! !\right]$, yielding the language $\mathcal{L}_{D E D L}$, whose formulas are generally denoted $\phi^{*}$ :

$$
\begin{gathered}
\mathcal{L}_{D E D L}^{\phi}: \phi::=p|\neg \phi| \phi \wedge \phi|B \phi| O \alpha|[\phi !] \phi|\left[\phi^{*} ! !\right] \phi \\
\mathcal{L}_{D E D L}^{\alpha}: \alpha::=\beta|\neg \alpha| \alpha \wedge \alpha|\alpha \wedge \phi| \phi \wedge \alpha\left|B^{\prime} \phi\right|[\phi !] \alpha \mid\left[\phi^{*} ! !\right] \alpha
\end{gathered}
$$

where $p$ ranges over $\Phi^{\phi}, \beta$ over $\Phi^{\alpha}$.

$[\psi !] \phi$ reads 'after learning $\psi, \phi$ holds', and $\left[\psi^{*} ! !\right] \phi$ reads 'after the promulgation/enforcement of $\psi^{*}, \phi$ holds'. Note that it is possible that $\psi^{*} \in \mathcal{L}_{E D L}^{\phi}$ because propositions can affect the normative realm via $R^{\prime}$. The semantics of these dynamic operators is inspired by Kooi [9] and defined as follows.

Definition 3. Let $M=\left(W, D, R, R^{\prime}, V\right)$ be an EDL-model, $\phi \in \mathcal{L}_{E D L}^{\phi}$ and $\psi^{*} \in \mathcal{L}_{E D L}$. We define the EDL-models $M * \psi$ ! and $M * \psi^{*} ! !$ as follows.

- $M * \psi !=\left(W, D, R !, R^{\prime}, V\right)$ where for all $w \in W$, $R !(w)=R(w) \cap\|\psi\|$.

- $M * \psi^{*} ! !=\left(W, D ! !, R, R^{\prime} ! !, V\right)$ where for all $w \in W$, $R^{\prime} ! !(w)=\left\{\begin{array}{l}R^{\prime}(w) \cap\left\|\psi^{*}\right\| \text { if } \psi^{*} \in \mathcal{L}_{E D L}^{\phi} \\ R^{\prime}(w)\end{array}\right.$

$D ! !(w)=\left\{\begin{array}{lr}D(w) \cap\left\|\psi^{*}\right\| \text { if } \psi^{*} \in \mathcal{L}_{E D L}^{\alpha} \text { and } M, w \models P \psi^{*} \\ D(w) & \text { otherwise. }\end{array}\right.$

where $\left\|\phi^{*}\right\|=\left\{v \in M \mid M, v \models \phi^{*}\right\}$. The truth conditions:

$$
\begin{aligned}
& M, w=[\psi !] \phi^{*} \quad \text { iff } \quad M * \psi !, w=\phi^{*} \\
& M, w \models\left[\psi^{*} ! !\right] \phi^{*} \quad \text { iff } \quad M * \psi^{*} ! !, w \models \phi^{*} .
\end{aligned}
$$

Just as for $\mathcal{L}_{E D L}$ and $\mathcal{L}_{D L}$, we can show that the language $\mathcal{L}_{D E D L}$ is actually 'equivalent' to the language $\mathcal{L}_{D E D L}^{\prime}=\mathcal{L}_{D E D L}^{\phi} \cup \mathcal{L}_{D E D L}^{\alpha^{\prime}}$ with 'pure' practitions $\mathcal{L}_{D E D L}^{\alpha^{\prime}}$ :

$$
\mathcal{L}_{D E D L}^{\alpha^{\prime}}: \alpha::=\beta|\neg \alpha| \alpha \wedge \alpha\left|B^{\prime} \phi\right|[\phi !] \alpha \mid\left[\phi^{*} ! !\right] \alpha
$$

where $p$ ranges over $\Phi^{\phi}$ and $\beta$ over $\Phi^{\alpha}$.

Proposition 3. Let $\phi \in \mathcal{L}_{D E D L}$. There is $\phi^{\prime} \in \mathcal{L}_{D E D L}^{\prime}$ such that $\models \phi \leftrightarrow \phi^{\prime}$.

In other words, instead of dealing with formulas of $\mathcal{L}_{D E D L}$, we could equivalently deal only with formulas of $\mathcal{L}_{D E D L}^{\prime}$. 


\section{$3.2 \quad$ Examples}

Changing beliefs: $[\phi !]$. Our logic is a dynamic epistemic logic, which allows to express communicative acts changing the beliefs of agents.

Example 2. Let us take up Example1, A colleague of the librarian informs him that the journal is actually on the list of journals free of charge for the library because the university subscribed to this journal.

$$
M, w=J \text { InList } \wedge[\text { JinList! }] \text { BJInList }
$$

After the communicative act the librarian knows that the journal is on the list of journals for which the library has free access. The resulting situation is depicted in Figure 2, To evaluate the formula [JInList!]BJInList in $M, w$ it is necessary to move to $M * J$ InList! (illustrated in Figure 2) and evaluate BJInList. All worlds where $J$ InList is false are not accessible anymore via the $R$ relation. In particular, from the real world $w$ only itself is accessible via $R$.

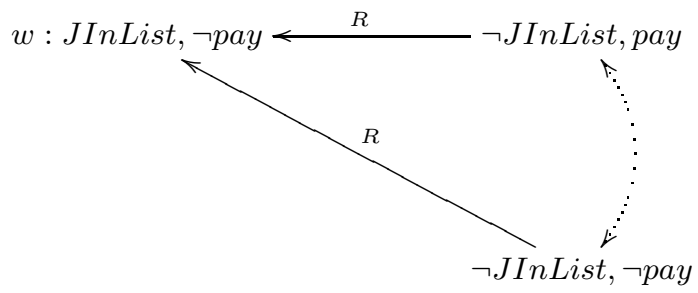

Fig. 2. Update by JInList!

Changing norms: $[\phi ! !]$. Our logic is a dynamic deontic logic, which allows to express communicative acts changing the norms.

Example 3. Let us take up Example 1 again. The Springer web-site declares that this journal is now free of charge for the library since today to fulfil the newly stipulated contract. This event can be modeled by the communicative act $[\neg$ pay!!]:

$$
M, w \models[\neg p a y ! !](O \neg p a y \wedge B O \neg p a y)
$$

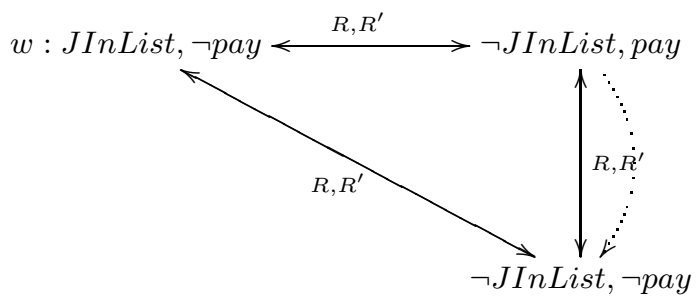

Fig. 3. Update by $\neg$ pay!! 
After this communicative act, one should not pay to download any paper of the journal and the librarian knows this (it is not possible to access from an $R$ world a world via $D$ where pay is true). This resulting situation is depicted in Figure 3. The only difference with the $E D L$-model of Figure 1 is that there is no dotted arrow from the bottom world to the top world on the right. This modification ensures that the only $D$ world where pay is true is not accessible anymore.

\section{How to Model Prescriptive and Descriptive Obligations}

Alchourrón and Bulygin [12] discussed the possibility of a logic of norms, which they distinguish from the logic of normative propositions. Alchourrón explains the distinction with the following box metaphor.

"We may depict the difference between the descriptive meaning (normative propositions) and the prescriptive meaning (norm) of deontic sentences by means of thinking the obligatory sets as well as the permitted sets as different boxes ready to be filled. When the authority $\alpha$ uses a deontic sentence prescriptively to norm an action, his activity belongs to the same category as putting something into a box. When $\alpha$, or someone else, uses the deontic sentence descriptively his activity belongs to the same category as making a picture of $\alpha$ putting something into a box. A proposition is like a picture of reality, so to assert a proposition is like making a picture of reality. On the other hand to issue (enact) a norm is like putting something in a box. It is a way of creating something, of building a part of reality (the normative qualification of an action) with the purpose that the addressees have the option to perform the authorized actions while performing the commanded actions." [1]

In our logic we can distinguish Alchourrón's distinction between descriptive and prescriptive norms. We map this distinction to the context of agent communication. The descriptive communicative act of the Springer web-site announcing that he should not pay can be modeled by the communicative act $[O \neg$ pay!]. Note that informing about the existence of a norm can enable the audience to know more information: for example, if the librarian should not pay for downloading any paper of a journal then he knows that this journal is on the list of journals for which the library has free access. The prescriptive communicative act of the librarian being informed that the journal is now free of charge can be modeled by the communicative act $[\neg$ pay!!].

This mapping allows to understand the role of agent systems in deontic logic, since a traditional problem can be solved by stating it in terms of interaction among agents.

Example 4. Let us take up Example 1]. Concerning the descriptive character of norms, we model the action of communicating that there is a norm obliging not 
to pay to download any paper as the announcement of the obligation $O \neg p a y$ !. The resulting situation is the same as the one depicted in Figure 2, After such announcement to the librarian, not only he believes that he should not pay to download any paper of the journal but also that the journal is in the list of journals for which the library has free access:

$$
M, w \models \neg B J I n L i s t \wedge[O \neg p a y !](B O \neg p a y \wedge B J I n L i s t)
$$

The inference $[O \neg p a y !] B J I n L i s t$ is possible if one should not pay Springer to download any paper of a journal which is in the list:

$$
\text { JInList } \rightarrow O \neg \text { pay }
$$

Note that pay is a practition, since it is in the scope of a deontic operator.

Concerning the prescriptive character of obligation, we model the action of putting a norm into existence, for example, by the Springer web-site announcing that from now on the library should not pay to download any paper of the journal as the announcement of the practition $\neg$ pay!!. The resulting situation is depicted in Figure 3. Note that, in this case, even if JInList $\rightarrow O \neg$ pay, we cannot derive that the librarian knows that the journal is in the list. This is intuitively correct: the new norm has just been introduced today so there has not been enough time to update the list (it is updated every month).

$$
M, w \models \neg B J \text { InList } \wedge[\neg \text { pay!! }](O \neg \text { pay } \wedge \neg \text { BJInList }) .
$$

\section{Conclusions}

Distinguishing the prescriptive and descriptive use of language is a classical challenge from deontic logic with practical consequences. If one agent tells another agent that he is obliged to do something, but the second agent would like to disagree, then the second agent should know whether the agent is creating a norm for him, or whether he is describing an existing normative system. In the first case he may disagree by responding that the agent is not authorized to create obligations for him, in the second case he may argue that the norm does not apply to him, or that the norm does not exist. Several formal systems therefore distinguish between prescriptive and descriptive obligations, but thus far the distinction was not analyzed in more detail, and the two kinds of obligations were not related to each other in an integrated framework.

In this paper, we give a more detailed analysis by modeling besides the normative system also the epistemic states of the agents, and how norms can be changed over time. Few articles in deontic logic deal with the interaction among deontic and epistemic notions, though they often entertain a tight relationship. Citizens must often know their obligations, e.g., people should know that it is forbidden to speed. Moreover, some obligations hold only in an epistemic context, e.g., the librarian is obliged not to pay if he knows that the journal is on the free access list 12. To specify such examples of autonomous agents acting 
within a normative system, there is a need for the logical formalization of these relationships. To model the interaction between epistemic and normative notions in a dynamic setting we introduced a general Dynamic Epistemic Deontic Logic. The logic extends a simplified version of Castañeda's deontic logic of practitions and propositions with epistemic and dynamic update operators.

In [3] we adapt this Dynamic Epistemic Deontic Logic to the problem of privacy regulations, introducing the notion of permitted and obligatory announcement, and the notion of compliance. The extended framework can deal with a new version of the Chinese wall problem, meta-policies specifying if a user can know the privacy policies and it distinguishes between permissions and obligations to let the user know with respect to the permissions and obligations to communicate information by means of messages.

Further research concerns making the logic multi-agent, to study the implications of our approach for contrary to duties and deontic detachment.

\section{References}

1. Alchourrón, C.: Philosophical foundations of deontic logic and the logic of defeasible conditionals. In: Meyer, J.-J., Wieringa, R. (eds.) Deontic Logic in Computer Science: Normative System Specification, pp. 43-84. John Wiley \& Sons, Chichester (1993)

2. Alchourron, C.E., Bulygin, E.: The expressive conception of norms. In: Hilpinen, R. (ed.) New studies in deontic logic, pp. 95-124. D. Reidel, Dordrecht (1981)

3. Aucher, G., Boella, G., van der Torre, L.: Privacy regulations in dynamic epistemic deontic logic. In: Procs. of Non Monotonic Reasoning Workshop 2010 (2010)

4. Blackburn, P., de Rijke, M., Venema, Y.: Modal Logic. Cambridge Tracts in Computer Science, vol. 53. Cambridge University Press, Cambridge (2001)

5. Boella, G., Verhagen, H., van der Torre, L.: Introduction to the special issue on normative multiagent systems. Journal of Autonomous Agents and Multi Agent Systems, 1-10 (2008)

6. Castañeda, H.N.: The paradoxes of Deontic Logic: the simplest solution to all of them in one fell swoop, pp. 37-86. Synthese library (1981)

7. Castañeda, H.N.: Knowledge and epistemic obligation. Philosophical perspectives 2, 211-233 (1988)

8. Hansen, J.: Imperatives and deontic logic: On the semantic foundations of deontic logic. PhD thesis, University of Leipzig, Germany (2008)

9. Kooi, B.: Probabilistic dynamic epistemic logic. Journal of Logic, Language and Information 12(4), 381-408 (2003)

10. Makinson, D.: On a fundamental problem of deontic logic. In: McNamara, P., Prakken, H. (eds.) Norms, Logics and Information Systems. New Studies on Deontic Logic and Computer Science, pp. 29-54. IOS Press, Amsterdam (1999)

11. Makinson, D., van der Torre, L.: Input-output logics. Journal of Philosophical Logic 29(4), 383-408 (2000)

12. Pacuit, E., Parikh, R.: The logic of knowledge based obligation. Synthese 149(2) (2006) 
13. van der Torre, L., Tan, Y.: An update semantics for deontic reasoning. In: McNamara, P., Prakken, H. (eds.) Norms, Logics and Information Systems. New Studies on Deontic Logic and Computer Science, pp. 73-90. IOS Press, Amsterdam (1999)

14. von Wright, G.H.: On the logic of norms and actions. In: Hilpinen, R. (ed.) New Studies in Deontic Logic: Norms, Actions and the Foundations of Ethics, pp. 3-35. D.Reidel, Dordrechtz (1981)

15. von Wright, G.H.: Deontic logic - as I see it. In: McNamara, P., Prakken, H. (eds.) Norms, Logics and Information Systems. New Studies on Deontic Logic and Computer Science, pp. 15-25. IOS Press, Amsterdam (1999) 